\title{
ANALYTICAL INSTRUMENTATION AND TECHNOLOGY
}

\author{
ISSN 2500-316X (Online) \\ https://doi.org/10.32362/2500-316X-2020-8-6-130-142 \\ УДК 621.3.051.024
}

(cc) BY

\section{Учёт магнитного поля системы электроснабжения энергоёмкого технического объекта}

\section{A.T. Тарланов ${ }^{\circledR}$, \\ 3.М. Курбанисмаилов}

МИРЭА - Российский технологический университет, Москва 119454, Россия

${ }^{\circledR}$ Автор для переписки, е-таil: lev.brave@gmail.com

В работе показан подход и результат учета взаимного влияния бортовых подсистем сложного технического объекта по цепям питания постоянным током. Под техническим объектом в данном случае понимается мобильное энергоемкое средство такое, как летательный аппарат, надводное или подводное судно, или железнодорожный локомотив с сильными магнитными полями. Целью работы является создание простого и наглядного инструмента математического моделирования вектора магнитного поля в произвольно заданной точке наблюдения. Это решение позволит добиться повышения точности магнитных измерений на борту, в частности, в задачах навигации. Отмечены недостатки коммерческих программ аналогичного назначения. Описана привязка объектов рассмотрения к общей системе координат, показан и детально описан аналитический алгоритм вычисления вектора магнитного поля, создаваемого бортовой кабельной сетью, имеющей выраженную 3D-траекторию. Приведены примеры визуализации результатов моделирования. Для решения задачи использован алгоритм вычисления вектора индукции на основе закона Био - Савара. Рассмотрен конкретный силовой кабель бортовой сети, заданный набором прямолинейных проводников с током. Намечены пути совершенствования созданного алгоритма с переходом от одной точки наблюдения к карте поля в заданной трехмерной зоне произвольного положения, объема и ориентации. Полученный результат рассматривается как элемент процедуры достижения электромагнитной совместимости энергоемких и высокочувствительных подсистем современного сложного технического объекта. 
Ключевые слова: индукция, магнитное поле, бортовая кабельная сеть, технический объект, прямолинейный проводник.

Для цитирования: Тарланов А.Т., Курбанисмаилов 3.М. Учёт магнитного поля системы электроснабжения энергоёмкого технического объекта. Российский технологический журнал. 2020;8(6):130-142. https://doi.org/10.32362/2500-316X-2020-8-6-130-142

\title{
Accounting of the magnetic field of the power supply system of a power-capacitive technical object
}

\section{A.T. Tarlanov ${ }^{\circledR}$ \\ Z.M. Kurbanismailov}

\author{
MIREA - Russian Technological University, Moscow 119454, Russia \\ ${ }^{\circledR}$ Corresponding author, e-mail: lev.brave@gmail.com
}

The paper shows the approach and the result of taking into account the mutual influence of on-board subsystems of a complex technical object along the DC power supply circuits. Technical objects are understood as a mobile, energy-intensive vehicle, such as an aircraft, a surface or submarine vessel, or a railway locomotive with strong magnetic fields. The aim of the work is to create a simple and intuitive tool for mathematical modeling of the magnetic field vector at an arbitrarily specified observation point. The task is being solved in order to improve the accuracy of magnetic measurements on board, in particular, in navigation problems. On-board DC networks are considered, to which the approach of mathematical modeling is applied. The disadvantages of commercial programs of a similar purpose are noted. The binding of the objects under consideration to the general coordinate system is described. An analytical algorithm for calculating the magnetic field vector from the on-board cable network with a pronounced 3D trajectory is shown. Examples of visualization of the simulation results are given. An algorithm for calculating the induction vector based on the Biot-Savard law is considered. The algorithm for the analytical solution of the problem is described in detail. A specific power cable of the on-board network is considered. The cable is given by a set of straight conductors with current. The ways of future improvement of the created product with the transition from one observation point to the field map in a given three-dimensional zone of arbitrary position, volume and orientation are outlined. The obtained result is considered as an element of the procedure for achieving electromagnetic compatibility of energy-intensive and highly sensitive subsystems of a modern complex technical object.

Keywords: induction, magnetic field, on-board cable network, technical object, straight conductor.

For citation: Tarlanov A.T., Kurbanismailov Z.M. Accounting of the magnetic field of the power supply system of a power-capacitive technical object. Rossiiskii tekhnologicheskii zhurnal = Russian Technological Journal. 2020;8(6):130142 (in Russ.). https://doi.org/10.32362/2500-316X-2020-8-6-130-142 


\section{Введение}

$\mathrm{H}$ аучно-технический прогресс позволяет создавать все более сложные технические системы, содержащие большое число различных подсистем, влияющих друг на друга [1-6]. Совершенство одной из таких обособленных подсистем вовсе не дает ей права на благополучное функционирование в окружении других, не менее совершенных, из-за их взаимного влияния, в общем случае непрогнозируемого. Взаимное влияние различных устройств на борту технического объекта (ТО) составляет одну из актуальных проблем современного приборо- и машиностроения $[1,10]$. Решение этой проблемы видится, в том числе, и в создании арсенала средств для контроля и моделирования указанного влияния, которые позволяли бы в реальном времени верифицировать различные версии компоновки технического объекта на разных этапах его создания, начиная с разработки.

Целью настоящей работы является создание простого, интуитивно понятного и действующего в реальном времени инструмента для оценки влияния стационарного магнитного поля, создаваемого бортовыми кабельными сетями (БКС) на подсистемы объекта, расположенные в произвольной точке заданной системы координат. Это решение имеет прямое отношение к известной проблеме электромагнитной совместимости (ЭМС) бортовых систем, которая решается, в частности, средствами измерения и моделирования создаваемых полей.

Предложенный в данной работе программный продукт будет использоваться в общем потоке решений ЭМС, особенно на начальном этапе проработки концептуальных решений, в частности, по проектированию кабельных силовых соединений различных подсистем объекта как друг с другом, так и с централизованной системой энергообеспечения. Решаемыми задачами являются: математическое описание силового кабеля, нахождение алгоритма суммирования полей в точке наблюдения от разных элементов БКС, визуализация результатов вычислений.

В результате работы создан перспективный программный продукт, позволяющий качественно оценивать взаимное влияние различных подсистем ТО по цепям питания, который может найти применение в практике проектирования современных технических систем в таких областях, как авиаприборостроение, космическая техника, судостроение и другие.

\section{Подход к получению карты поля на борту}

Под техническим объектом будем понимать мобильное средство, снабженное как силовыми установками, приводящими его в движение, так и устройствами управления такими установками, подверженными влиянию внешних электромагнитных полей. К таким объектам без сомнения можно отнести летательные аппараты различного назначения, включая и космическую технику, надводные и подводные суда с мощными тяговыми электродвигателями и аккумуляторными батареями, а также железнодорожные локомотивы, где, как известно, сильны магнитные поля от силовых установок.

Наличие на борту такого ТО даже простого магнитного компаса уже требует контроля магнитных полей, создаваемых бортовыми системами, а при наличии многочисленных бортовых измерительных систем проблема ЭМС приобретает еще большую актуальность. В некоторых системах [17] прямо стоит задача знания точного распределения магнитного поля в заданной 3D-зоне, и количество таких проектов возрастает. 
Бортовые кабельные сети принято делить на сети переменного и постоянного тока. В данной работе рассматриваются постоянные магнитные поля, но результаты можно распространить и на сети переменного тока, которые, как правило, имеют большую протяженность, при меньшей величине тока.

Методы получения карты любого физического поля в заданных точках или в пределах зоны наблюдения принято делить на математические и физические. Картографирование поля, как этап физического моделирования, реализует аппаратное решение проблемы ЭМС, и применяется, как правило, на завершающих этапах создания технических объектов.

На этапе проектирования более актуальны математические методы моделирования, которым и посвящена настоящая работа. Кроме того, математическое моделирование обходится несравнимо дешевле физического, что особенно привлекательно на начальных этапах создания ТО. Математическое моделирование требует знания источников поля, а также привязки к системе координат объекта.

Известны коммерческие программные продукты математического моделирования магнитных полей, например, пакет ELCUT (https:/elcut.ru/). Однако, данный продукт и аналогичные продукты не позволяют проводить 3D-моделирование при произвольной траектории БКС. Будем искать простой аналитический метод математического моделирования стационарного магнитного поля в произвольной точке наблюдения, создаваемого произвольной 3D-траекторией бортовой кабельной сети постоянного тока.

\section{Методология учёта магнитного поля кабельных сетей на борту ТО}

Зададим декартову систему координат для моделирования магнитного поля на борту ТО, как показано на рис. 1.

Источниками магнитного поля являются кабели бортовой сети. Для описания прокладки кабелей на борту в рассматриваемой системе координат каждый из них представляется набором прямолинейных проводников с заданными координатами концов [3]. Величину индукции магнитного поля, создаваемой каждым кабелем в произвольно выбранной точке наблюдения, можно получить с помощью векторной суперпозиции индукций $[4,5]$ от элементарных прямолинейных отрезков кабелей.

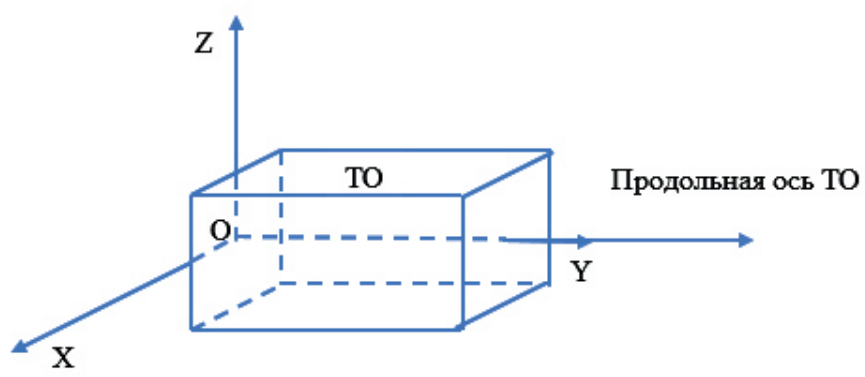

Рис. 1. Система координат для моделирования магнитного поля на борту ТО.

Индукция, создаваемая прямолинейным конечным проводником в удаленной точке наблюдения, определяется на основе закона Био - Савара - Лапласа [6], который в векторной форме имеет следующий вид:

$$
d \vec{B}=\frac{\mu_{0}}{4 \pi} \frac{[d \vec{l} \times \vec{r}]}{r^{2}},
$$


где $\mu_{0}=4 \pi \cdot 10^{-7}($ Гн $\cdot$ м) - магнитная постоянная; $I-$ сила тока в проводнике $(\mathrm{A}) ; d \vec{l}-$ элемент проводника; $\vec{r}$ - радиус-вектор направления из центра проводника на удалённую точку наблюдения; $r$ - модуль радиус-вектора (м).

Модуль вектора индукции $[7,8]$ определяется формулой:

$$
d B=\frac{\mu_{0}}{4 \pi} \frac{d l \sin \alpha}{r^{2}}
$$

где $\alpha$ - угол между элементом проводника и радиус-вектором.

Схема взаимного расположения точки наблюдения и прямолинейного проводника с током показана на рис. 2. Так как проводник имеет конечную длину, то угол изменяется от значения $\alpha_{1}$ в точке $A$ - начале рассматриваемого проводника - до значения $\alpha_{2}$ в точке $B$ - конце рассматриваемого проводника, что задает пределы интегрирования формулы (2) для поиска общего значения модуля индукции, создаваемой всем проводником в точке наблюдения $C$ [9].
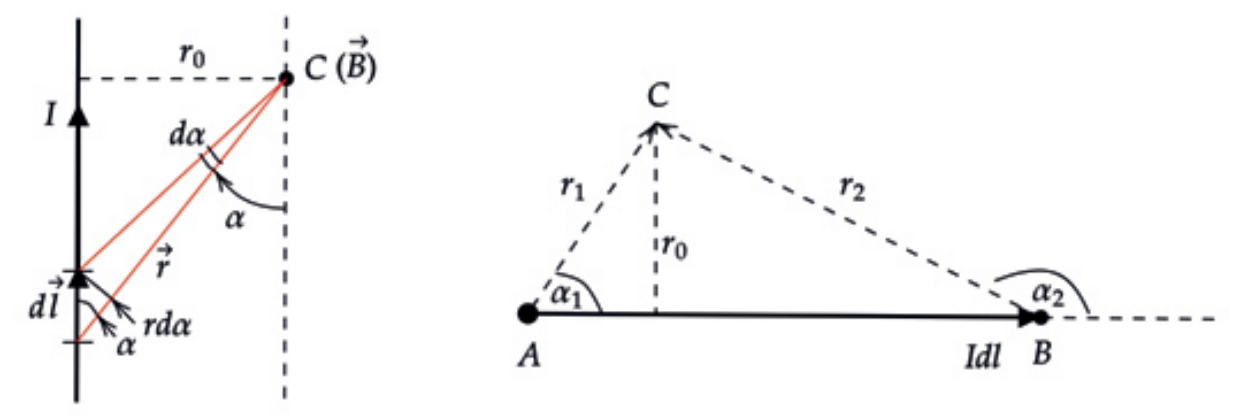

Рис. 2. Расположение точки наблюдения $C$ относительно конечного прямолинейного проводника $A B$.

Из рис. 2 видно, что

$$
r=\frac{r_{0}}{\sin \alpha}
$$

где $r_{0}$ - расстояние от наблюдаемой точки $C$ до прямой, содержащей отрезок $A B$.

Получаем:

$$
d l=r \frac{d \alpha}{\sin \alpha}=r_{0} \frac{d \alpha}{\sin ^{2} \alpha}
$$

Далее подставляем (4) в формулу (2) и получаем:

$$
d B=\frac{\mu_{0}}{4 \pi} \frac{l}{r_{0}} \sin \alpha d \alpha
$$

Интегрируя выражение (5) по углу $\alpha$ в пределах от $\alpha_{1}$ до $\alpha_{2}$, получаем расчётную формулу:

$$
B=\frac{\mu_{0}}{4 \pi} \frac{l}{r_{0}}\left(\cos \alpha_{1}-\cos \alpha_{2}\right) \text {. }
$$

Далее переходим к заданию целого кабеля в виде набора прямолинейных проводников (ПП). Для каждого ПП задаются два набора координат XYZ - для начала (А) и конца (B). Начало и конец каждого ПП выбираются в соответствии с действующей конструкцией кабельных соединений на борту ТО. Задание координат ПП целесообразно представить в виде таблицы. 
Здание координат ПП

\begin{tabular}{c|c|c|c|c|c|c}
\hline \multirow{2}{*}{ Номер ПП } & \multicolumn{3}{|c|}{ Начало $(A)$} & \multicolumn{3}{c}{ Конец $(B)$} \\
\cline { 2 - 7 } & $X_{1}$ & $Y_{1}$ & $Z_{1}$ & $X_{2}$ & $Y_{2}$ & $Z_{2}$ \\
\hline 1 & 1.00 & 1.50 & 2.00 & 1.00 & 2.00 & 0.50 \\
\hline 2 & 1.00 & 2.00 & 0.50 & -0.50 & 3.00 & -0.70 \\
\hline 3 & -0.50 & 3.00 & -0.70 & -0.50 & 3.50 & -0.70 \\
\hline 4 & -0.50 & 3.50 & -0.70 & 1.00 & 4.00 & 0.00 \\
\hline 5 & 1.00 & 4.00 & 0.00 & 0.00 & 4.50 & 1.00 \\
\hline 6 & 0.00 & 4.50 & 1.00 & 0.00 & 4.70 & 1.50 \\
\hline 7 & 0.00 & 4.70 & 1.50 & 1.00 & 4.70 & 1.50 \\
\hline 8 & 1.00 & 4.70 & 1.50 & 0.00 & 4.90 & 2.00 \\
\hline
\end{tabular}

Из данных таблицы можно определить геометрию единого кабеля, состоящего из 8 отдельных ПП. Как видно, конец предыдущего ПП совпадает с началом следующего.

Таким же образом задаются координаты скользящей точки наблюдения $C$, например $X_{3}=-2, Y_{3}=0, Z_{3}=-2$ (м) в принятой системе координат.

Далее, пользуясь построениями на рис. 2, можно для каждого заданного ПП вычислить характеристики треугольника $A C B$, длины сторон которого определяются по формуле Пифагора:

$$
A B=\sqrt{\left(X_{2}-X_{1}\right)^{2}+\left(Y_{2}-Y_{1}\right)^{2}+\left(Z_{2}-Z_{1}\right)^{2}} .
$$

Аналогично вычисляются и длины других сторон.

Следующий шаг - вычисление косинусов необходимых для определения углов треугольника. Например, для угла $C A B$ (он же $\alpha_{1}$, вычисляем косинус угла между векторами $A B$ и $A C$ :

$$
\cos \alpha_{1}=\frac{\left(X_{3}-X_{1}\right)\left(X_{2}-X_{1}\right)+\left(Y_{3}-Y_{1}\right)\left(Y_{2}-Y_{1}\right)+\left(Z_{3}-Z_{1}\right)\left(Z_{2}-Z_{1}\right)}{A B \cdot A C} .
$$

Вычитание координат точки $A$ в числителе согласовывает параллельный перенос векторов в начало координат $[10,11]$. Аналогично вычисляется косинус угла $A B C$ (он же $\left.180^{\circ}-\alpha_{2}\right)$ и далее преобразованием:

$$
\cos \left(180^{\circ}-\arccos (A B C)\right)
$$

получаем искомый $\cos \alpha_{2}$.

Затем, задавая значение тока $I$ в кабеле, найдем величину магнитной индукции $B(6)$, создаваемой каждым ПП рассматриваемого кабеля [12]. Для получения суммарной индукции в точке $C$ необходимо выполнить векторное сложение индукций от каждого ПП в этой точке [13].

Магнитное поле, создаваемое каждым ПП в точке наблюдения $C$, имеет направление, перпендикулярное к плоскости, в которой лежат ПП и точка $C$, по правилу «правого винта», с учетом направления тока в ПП [14].

Для вычисления направления полного вектора необходимо найти векторное произведение векторов $\overrightarrow{A B}$ и $\overrightarrow{A C}$. Для этого рассчитывается определитель трёхмерной матрицы: 


$$
\overrightarrow{A B} \cdot \overrightarrow{A C}=\vec{V}=\left|\begin{array}{ccc}
i & j & k \\
X_{2}-X_{1} & Y_{2}-Y_{1} & Z_{2}-Z_{1} \\
X_{3}-X_{1} & Y_{3}-Y_{1} & Z_{3}-Z_{1}
\end{array}\right|
$$

Для расчёта определителя и получения координат вектора $\vec{V}$, определенного формулой (9), используются следующие формулы:

$$
\begin{aligned}
& X_{V}=\left(Y_{2}-Y_{1}\right)\left(Z_{3}-Z_{1}\right)-\left(Z_{2}-Z_{1}\right)\left(Y_{3}-Y_{1}\right) ; \\
& Y_{V}=\left(Z_{2}-Z_{1}\right)\left(X_{3}-X_{1}\right)-\left(X_{2}-X_{1}\right)\left(Z_{3}-Z_{1}\right) ; \\
& Z_{V}=\left(X_{2}-X_{1}\right)\left(Y_{3}-Y_{1}\right)-\left(Y_{2}-Y_{1}\right)\left(X_{3}-X_{1}\right) .
\end{aligned}
$$

Длина вектора $\vec{V}$, определяется формулой Пифагора:

$$
|V|=\sqrt{X_{V}^{2}+Y_{V}^{2}+Z_{V}^{2}} .
$$

Если нормировать каждую координату вектора $\vec{V}\left(X_{V}, Y_{V}, Z_{V}\right)$ на его длину $|V|$, то получатся направляющие косинусы, которые достаточно умножить на скалярную величину магнитной индукции $B$ [15], чтобы получить координаты вектора магнитной индукции, создаваемого каждым конкретным ПП:

$$
\begin{aligned}
& X_{B \Pi \Pi}=\frac{X_{V}}{|V|} B ; \\
& Y_{B \Pi \Pi}=\frac{Y_{V}}{|V|} B ; \\
& Z_{B \Pi \Pi}=\frac{Z_{V}}{|V|} B .
\end{aligned}
$$

Арифметическая сумма всех соответствующих координат для каждого ПП даёт координату вектора $B$ :

$$
\begin{aligned}
& X_{B}=\sum X_{B \Pi \Pi} \\
& Y_{B}=\sum Y_{B \Pi \Pi} \\
& Z_{B}=\sum Z_{B \Pi \Pi} .
\end{aligned}
$$

Соответственно, длина этого суммарного вектора магнитной индукции $B$, отображающая абсолютное значение общей магнитной индукции от всего кабеля в точке $C$, определяется формулой:

$$
B=\sqrt{X_{B}^{2}+Y_{B}^{2}+Z_{B}^{2}}
$$

При наличии других кабелей, заданных аналогичным образом, для нахождения суммарной индукции в точке $C$ такие же вычисления проводятся для каждого кабеля, а их суммарная индукция определяется сложением векторов $B$ (арифметическая сумма соответствующих координат [16] с нахождением модуля результирующего вектора по формуле Пифагора. 
Точка $C$ названа скользящей в том смысле, что работа программы сводится к последовательному изменению координат точки наблюдения в заданных пределах перемещения, по заданной траектории и с заданным шагом. Результатом расчетов является карта индукции магнитного поля в заданной зоне наблюдения.

\section{Результаты и их обсуждение}

Попробуем реализовать на практике методологию учета магнитного поля для отдельного кабеля и составим карту индукции магнитного поля.

На рис. 3 показана геометрическая модель кабеля, построенная по данным таблицы, приведенной выше. Разместим произвольно на модели точку наблюдения $C$, например, с координатами: $X=0, Y=3, Z=0.5(\mathrm{M})$.

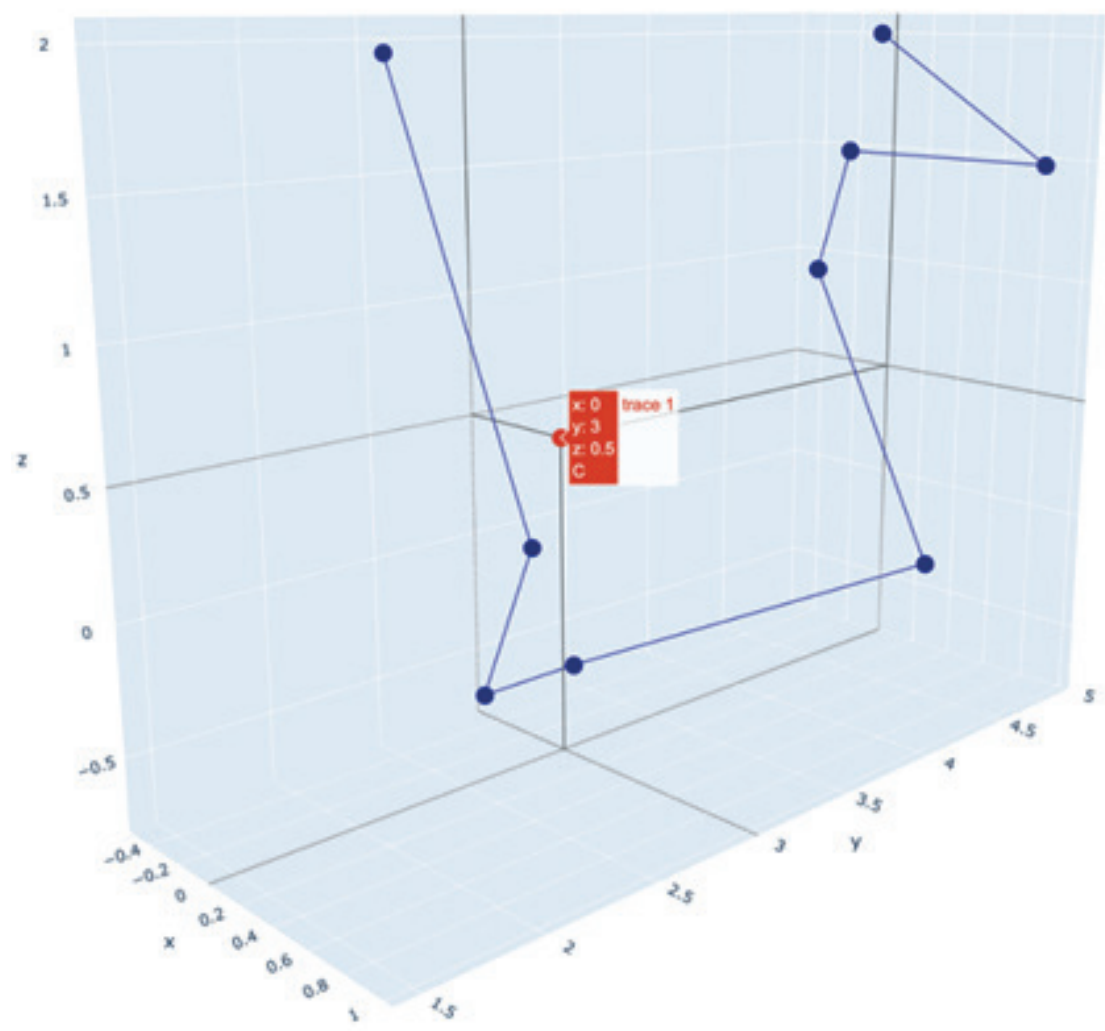

Рис. 3. Геометрическая модель рассматриваемого кабеля бортовой электропроводки ТО с обозначенной точкой наблюдения $C$.

Рассчитаем по описанной выше методике индукцию магнитного поля, создаваемого описанным кабелем в точке $C$ для величины тока $I=2$ А во всех последовательно включенных элементах ПП.

Влияние элементов конструкции ТО на распределение магнитного поля на данном этапе не учитываем. Результат расчета дает величину модуля общего вектора магнитной индукции в заданной точке наблюдения $C$, созданного всеми элементами силового кабеля

$$
B=6.22 \times 10^{-7} \text { Тл. }
$$

Представленная выше математическая модель позволяет анализировать величину модуля индукции магнитного поля в произвольно заданной точке наблюдения при произвольно заданном числе элементов тока вдоль силового кабеля. 
Для наблюдения за динамикой изменения силы магнитного поля в пространстве вокруг ТО необходимо произвести расчёт по большему количеству точек. Поэтому предложено формировать область, размеры которой строятся относительно максимальных и минимальных значений размеров ТО по осям. После этого данная область делится на равные части по каждой из трех осей $X Y Z$, образуя расчетную сетку из точек в пространстве с фиксированным шагом. Для примера создадим расчетную стеку из 1000 точек, по 10 точек на ось (рис. 4).

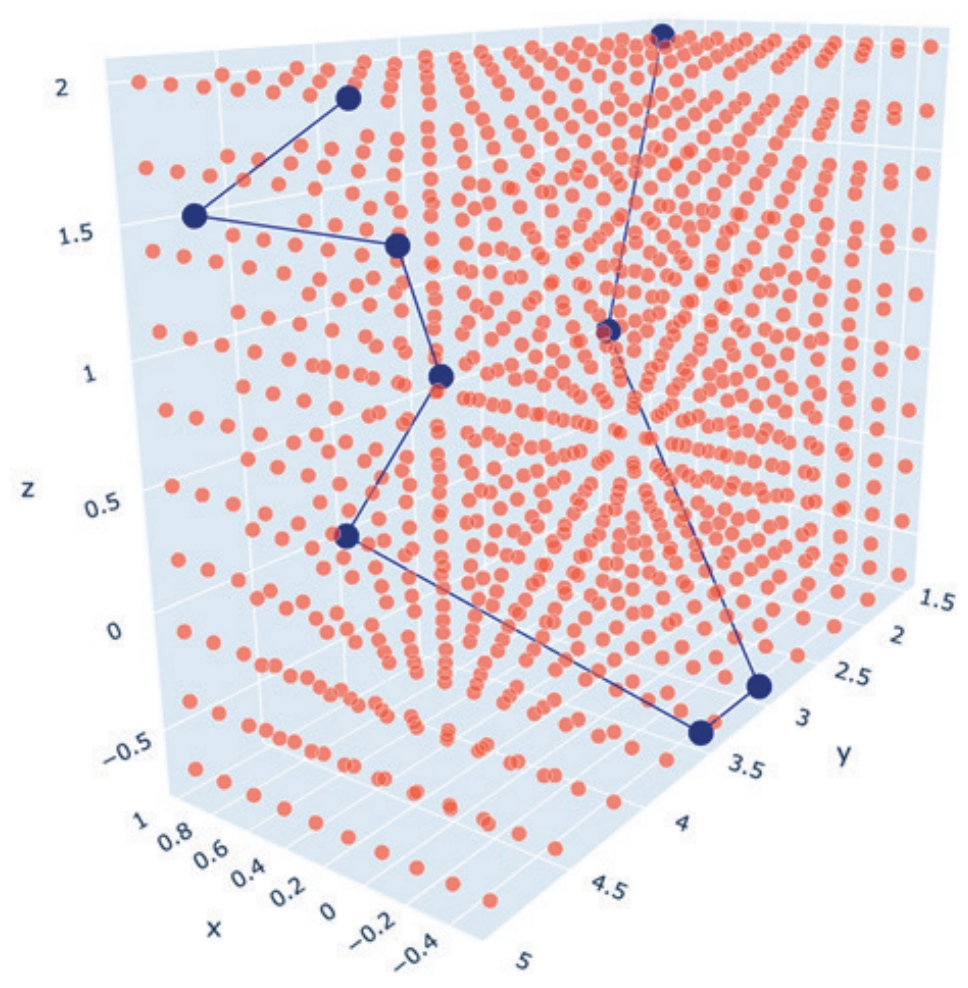

Рис. 4. Геометрическая модель рассматриваемого кабеля бортовой электропроводки ТО с расчетной сеткой из 1000 точек.

Расчет по предложенной математической модели производится для каждой отдельной точки из расчетной сетки (рис. 5).

На рис. 5 изображена так называемая точечная визуализация магнитного поля с использованием цвета как показателя величины модуля индукции магнитного поля (чем выше значение, тем цвет «теплее»). Для данного примера характерна одна «красная» точка с наивысшим значением индукции магнитного поля

Точечная визуализация имеет также и недостаток - при высоком разрешении расчетной сетки визуализируемый участок становится перенасыщен точками, которые имеют крайне малые значения. Это приводит к тому, что наблюдателю затруднительно определить необходимый участок расчётной сетки. Исходя из вышесказанного, целесообразно не отображать точки, значение модуля индукции которых стремится к нулю. Более того, на основе оставшихся точек можно визуализировать формируемое ими значимое поле, которое, по сути, формирует геометрическую форму, где вершины совпадают в пространстве с вычисленными значимыми точками и соединены между собой ребрами. Три значимые точки формируют плоскость. Промежуточные значения, которые лежат на ребрах или плоскостях, вычисляются с помощью линейной интерполяции между со- 
седними точками, значение модуля индукции в которых уже рассчитано. Таким образом вокруг рассчитанных значимых точек формируется «аура напряженности», вписанная в вычисляемый объем, что упрощает исследование и анализ результата.

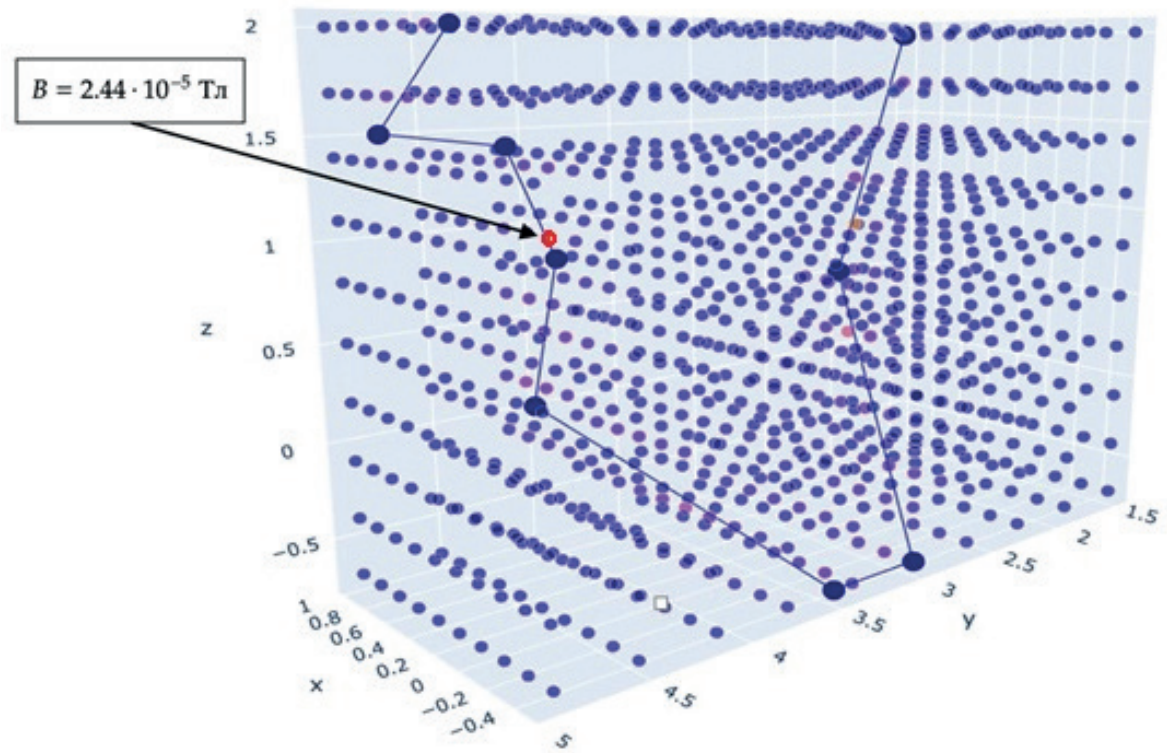

Рис. 5. Визуальное отображение магнитного поля по расчетной сетке из 1000 точек.

На рис. 6 приведена визуализация аналогичных результатов расчета модуля индукции для 1000 точек, как и на рис. 5, но с использованием линейной интерполяции.

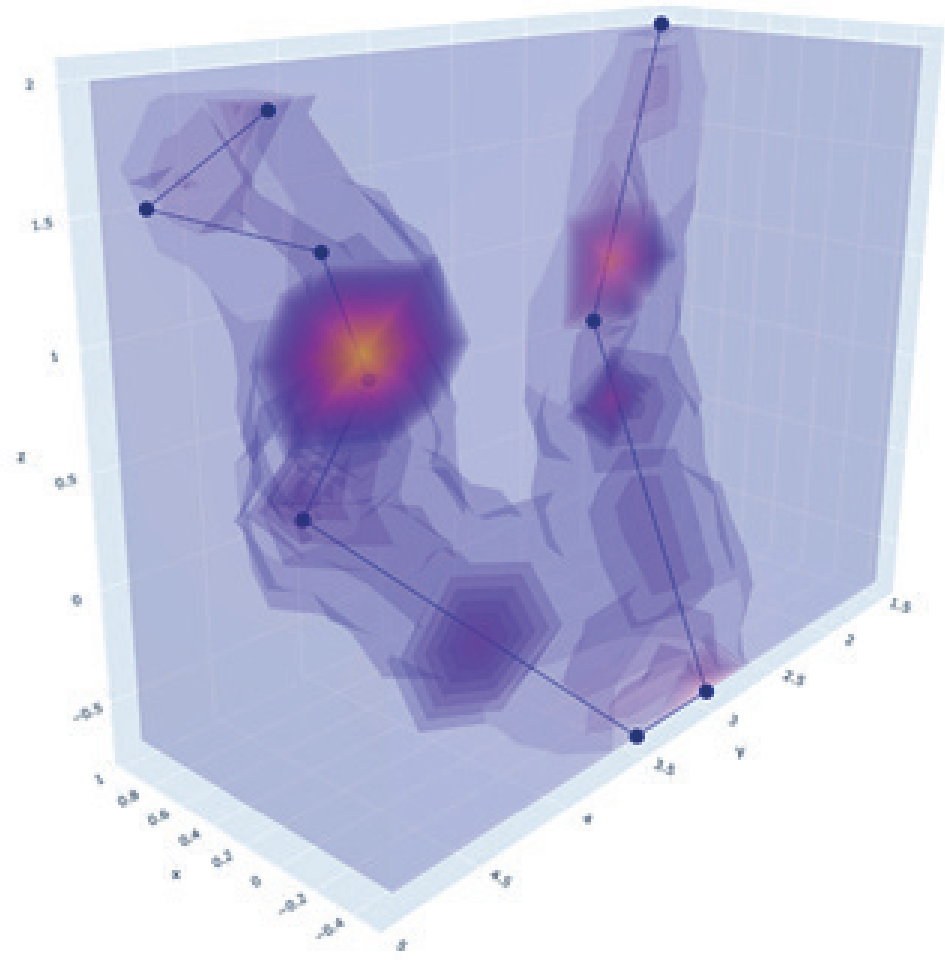

Рис. 6. Визуальное отображение магнитного поля по сетке из 1000 точек с использованием линейной интерполяции 


\section{Выводы}

Создан простой и наглядный алгоритм, позволяющий в реальном времени, оценивая результаты измерений, моделировать магнитное поле силовых цепей технического объекта в заданной точке трехмерного пространства зоны наблюдения. Алгоритм позволяет эффективно оценивать взаимное влияние друг на друга энергоёмких и высокочувствительных подсистем современного технического объекта. Авторам неизвестны алгоритмы с аналогичными свойствами. Наиболее эффективным видится применение предложенной математической модели на этапе проектирования, а также при проведении замены и модернизации оборудования бортовых систем.

В последующих работах будет также учтён эффект обратных проводов в БКС, которые, как правило, выполняются бифилярными для уменьшения полей рассеяния, что потребует повышения чувствительности измерений и моделирования.

Созданный алгоритм и программный продукт на его базе могут найти применение при разработке сложных бортовых систем современных подвижных платформ, содержащих энергоемкие системы движения, которые создают энергичные поля рассеяния, требующие учета в ходе проектирования, например, при разработке бортовой системы обеспечения современного авиационного лайнера типа SS-100.

\section{Источники финансирования}

Научно-исследовательская работа, результаты которой изложены в статье, выполнена за счет средств Централизованного фонда по теме «Система расчета электромагнитной совместимости бортовой кабельной сети на техническом объекте» на основании протокола заседания экспертной комиссии по подведению итогов Всероссийского конкурса «Инноващии в реализации приоритетных направлений развития науки и технологий» от 29 мая 2020 г., шифр - НИЧ ИЦМР 19/2020.

\section{Литература:}

1. Кузнецов А.В. Элементарная электротехника. М.: ДМК-Пресс; 2018. 700 с. ISBN 978-5-97060-577-6

2. Попов Н.А. Движение заряженных частиц в электрических и магнитных полях. М.: Прометей; 2015. 48 с. ISBN 978-5-99058-869-1

3. Коршунова Л.Н. Магнитные явления. Магнитная индукция. Сила Ампера. Сила Лоренца. Электромагнитная индукция. М.: Контур-М; 2005. 95 с. ISBN 5-98642-013-6

4. Каку М. Физика будущего. М.: Альпина нон-фикшн; 2012. 582 с. ISBN: 978-5-91671-164-6

5. Распространение радиоволн, под ред. О.И. Яковлева. М.: URSS: ЛЕНАНД; 2009. 491 с. ISBN 978-5-9710-0183-6

6. Планк М. Введение в теоретическую физику. Часть 3. Теория электричества и магнетизма. М.-Л.: ГТТИ, 1933.

7. Henry W. Electromagnetic Compatibility Engineering. Wiley; 2009. 880 p. ISBN 978-0470189306

8. Алешкевич В.А. Электромагнетизм. М.: ФИЗМАТЛИТ; 2014. 404 с. ISBN 978-5-9221-1555-1

9. Weston D.A. Electromagnetic Compatibility: Methods, Analysis, Circuits, and Measurement, Third Edition. CRC Press; 2016. 1184 p. ISBN 978-1482299502

10. Детлаф А.А., Яворский Б.М., Милковская Л.Б. Курс физики. Т. 2. Электричество и магнетизм (4-е издание). М.: Высшая школа; 1977. 375 с.

11. Джексон Дж. Классическая электродинамика. М.: Мир; 1965. 702 с.

12. Железняков В.В. Электромагнитные волны в космической плазме. Генерация и распространение. М.: Наука; 1977. 432 с.

13. Паршаков А.Н. Электромагнетизм в ключевых задачах. М.: Интеллект; 2019. 288 с. ISSN 978-5-91559-269-7

14. Попов А.Г. Движение заряженных частиц в электрических и магнитных полях. М.: Прометей; 2015. 46 c. ISBN 978-5-9905886-9-1 
15. Бондарев Б.В., Калашников Н.П., Спирин Г.Г. Курс общей физики: в 3 кн. Книга 2. Электромагнетизм. Волновая оптика. Квантовая физика. М.: Изд-во Юрайт; 2019. 441 с. ISBN 978-5-9916-1754-3

16. Williams T. EMC for Product Designers. Newnes, 2016. 552 p. ISBN 978-0081010167

17. Blood E.B. Device for quantitatively measuring the relative position and orientation of two bodies in the presence of metals utilizing direct current magnetic fields: Pat. 4945305 US. Appl. № US07/336,342. Publ. 31.07.1990.

\section{References:}

1. Kuznetsov A.V. Elementarnaya elektrotekhnika (Elementary electrical engineering). Moscow: DMK-Press; 2018. 700 p. (in Russ.). ISBN 978-5-97060-577-6

2. Popov N.A. Dvizhenie zaryazhennykh chastits v elektricheskikh i magnitnykh polyakh (The movement of charged particles in electric and magnetic fields). Moscow: Prometei; 2015. 48 p. (in Russ.). ISBN 978-5-99058-869-1

3. Korshunova L.N. Magnitnye yavleniya. Magnitnaya induktsiya. Sila Ampera. Sila Lorentsa. Elektromagnitnaya induktsiya (Magnetic phenomena. Magnetic induction. Ampere Force. Lorentz Force. Electromagnetic induction). Moscow: Kontur-M; 2005. 95 p. (in Russ.). ISBN 5-98642-013-6

4. Kaku M. Fizika budushchego (Physics of the future). Moscow: Al'pina non-fikshn; 2012. 582 p. (in Russ.). ISBN: 978-5-91671-164-6

[Kaku M. Physics of the future. New York: Doubleday; 2011. ISBN: 978-0-385-53080-4]

5. Rasprostranenie radiovoln (Propagation of radio waves); (Ed.) O.I. Yakovlev. Moscow: URSS: LENAND; 2009. 491 p. (in Russ.). ISBN 978-5-9710-0183-6

6. Plank M. Vvedenie v teoreticheskuyu fiziku. Chast' 3. Teoriya elektrichestva i magnetizma (Introduction to Theoretical Physics. Part 3. The theory of electricity and magnetism). Moscow- Leningrad: GTTI; 1933. 184 p. (in Russ.).

7. Henry W. Electromagnetic Compatibility Engineering. Wiley; 2009. 880 p. ISBN 978-0470189306

8. Aleshkevich V.A. Jelektromagnetizm (Electromagnetism). Moscow: FIZMATLIT; 2014. 404 p. (in Russ.). ISBN 978-5-9221-1555-1

9. Weston D.A. Electromagnetic Compatibility: Methods, Analysis, Circuits, and Measurement, Third Edition. CRC Press; 2016. 1184 p. ISBN 978-1482299502

10. Detlaf A.A., Yavorskii B.M., Milkovskaya L.B. Kurs fiziki. T. 2. Elektrichestvo i magnetizm (4-e izdanie). (Physics course. V. 2. Electricity and magnetism (4th edition)). Moscow: Vysshaya shkola; 1977. 375 p. (in Russ.).

11. Jackson J. Klassicheskaya elektrodinamika. (Classical electrodynamics). Moscow: Mir; 1965. 702 p. (in Russ.).

12. Zheleznyakov V.V. Elektromagnitnye volny v kosmicheskoi plazme. Generatsiya i rasprostranenie (Electromagnetic waves in cosmic plasma. Generation and distribution). Moscow: Nauka; 1977. 432 p. (in Russ.).

13. Parshakov A.N. Elektromagnetizm v klyuchevykh zadachakh (Electromagnetism in key tasks). Moscow: Intellekt; 2019. 288 p. (in Russ.). ISSN 978-5-91559-269-7

14. Popov A.G. Dvizhenie zaryazhennykh chastits v elektricheskikh i magnitnykh polyakh (The movement of charged particles in electric and magnetic fields). Moscow: Prometei; 46 p. (in Russ.). ISBN 978-5-9905886-9-1

15. Bondarev B.V., Kalashnikov N.P., Spirin G.G. Kurs obshchei fiziki: v 3 kn. Kniga 2. Elektromagnetizm. Volnovaya optika. Kvantovaya fizika (Electromagnetism. Wave optics. The quantum physics). Moscow: Izdatel'stvo Yurait; 2019. 441 p. (in Russ.). ISBN 978-5-9916-1754-3

16. Williams T. EMC for Product Designers. Newnes, 2016. 552 p. ISBN 978-0081010167

17. Blood E.B. Device for quantitatively measuring the relative position and orientation of two bodies in the presence of metals utilizing direct current magnetic fields: Pat. 4945305 US. Appl. № US07/336,342. Publ. 31.07.1990.

\section{Об авторах:}

Тарланов Арслан Тарланович, преподаватель кафедры КБ-4 «Интеллектуальные системы информационной безопасности» Института комплексной безопасности и специального приборостроения ФГБОУ ВО «МИРЭА - Российский технологический университет» (119454, Россия, Москва, пр-т Вернадского, д. 78). http://orcid.org/0000-0002-7508-9682

Курбанисмаилов Заур Магомедович, преподаватель кафедры КБ-4 «Интеллектуальные системы информационной безопасности» Института комплексной безопасности и специального приборостроения ФГБОУ ВО «МИРЭА - Российский технологический университет» (119454, Россия, Москва, пр-т Вернадского, д. 78). http://orcid.org/0000-0002-5388-049X 
About the authors:

Arslan T. Tarlanov, Lecturer, Department of "Intelligent Information Security Systems" of the Institute for Integrated Security and Special Instrument Engineering MIREA - Russian Technological University (78, Vernadskogo pr., Moscow 119454, Russia). http://orcid.org/0000-0002-7508-9682

Zaur M. Kurbanismailov, Lecturer, Department of "Intelligent Information Security Systems" of the Institute for Integrated Security and Special Instrument Engineering MIREA - Russian Technological University (78, Vernadskogo pr., Moscow 119454, Russia). http://orcid.org/0000-0002-5388-049X

Поступила: 25.05.2020; получена после доработки: 16.09.2020; принята к опубликованию: 30.09.2020. 